\title{
Directive pour les guides de pratique médicale
}

Depuis leur publication dans le Bulletin des médecins suisses [1], les propositions du Comité central ont été longuement et intensément discutées par les sociétés de discipline médicale (SDM). Les avis positifs et négatifs ont été aussi nombreux les uns que les autres. Il convient cependant de souligner une nouvelle fois que le phénomène des guides de pratique médicale ne peut pas être freiné et que, par conséquent, la FMH doit agir. Cela dit, elle n'a nullement l'intention de s'immiscer dans l'activité des sociétés de discipline médicale, car les compétences nécessaires en ce qui concerne les quelque 50 disciplines médicales lui font défaut. Il s'agit cependant d'aboutir à une unification de la forme, du mode d'élaboration et des critères des guides de pratique médicale, afin de rendre leur utilisation plus aisée. C'est pourquoi, le groupe de travail «Guides de pratique» a élaboré des recommandations. En outre, la liste de contrôle publiée ci-après donne des indications quant aux critères de qualité pris en considération par le groupe de travail pour la reconnaissance des guides; elle peut donc également servir aux SDM comme modèle pour l'élaboration de leurs guides de pratique.

Le domaine "Qualité» de la FMH se tient à disposition des sociétés de discipline médicale pour les assister dans l'élaboration des guides de pratique. En guise de conclusion, nous rappelons une nouvelle fois que les sociétés de discipline médicale sont invitées à soumettre leurs éventuelles propositions de modifications ou de compléments à propos de la directive pour les guides de pratique médicale jusqu'au 31 janvier 2000.

E. Eicher, membre du Comité central de la FMH, chargé du domaine "Qualité»

1 Directive pour les guides de pratique médicale. Bull méd suisses 1999;80(14):831-3.

\section{Recommandations} concernant la directive pour les guides de pratique médicale

Introduction, préambule ou autre

Remarques générales favorisant la compréhension du guide de pratique médicale.

Introduction liée au thème

Pertinence! Définition du problème.

\section{But des recommandations}

Remarque précisant s'il s'agit d'un guide de pratique diagnostique, thérapeutique, médico-technique ou autre. Dans la mesure du possible, indiquer le(s) code(s) CIM (classification internationale des maladies) concerné(s).

Méthode appliquée pour l'élaboration des guides de pratique

Par ex.:

- groupe de travail

- recherche bibliographique

- projet du groupe de travail

- processus consensuel et test clinique

- indications quant au degré de certitude scientifique des assertions du guide de pratique médicale (cf. littérature "Groupe d'étude canadien sur les soins de santé préventifs», dans la directive pour les guides de pratique médicale)

- prise en considération de la $1^{\text {re }}$ page de la liste de contrôle de la Société suisse de gynécologie et obstétrique (SSG0).

Recommandations proprement dites

En quelques phrases claires et précises, établir le libellé des recommandations. Si possible sur deux pages de format A4 pouvant être séparées du reste du guide de pratique.

Motifs des recommandations

L'argumentation détaillée de chaque assertion doit mentionner les preuves tirées de la littérature ou indiquer si l'assertion est simplement le fruit d'un consensus.

Les cas d'exception prévisibles doivent être mentionnés.

Les indications relatives à la diffusion et à la mise en œuvre du guide de pratique ainsi qu'à l'évaluation de son application doivent y figurer.

Le délai de mise à jour et les modalités d'abrogation doivent y figurer.

Il convient d'indiquer si un document destiné au patient et consacré au même thème est prévu.

Annexe

Explications complémentaires, accompagnées éventuellement de citations et d'extraits tirés de la littérature scientifique ainsi que de tableaux et de représentations graphiques, etc.

Bibliographie complète

Numérotation se référant au texte du guide de pratique. 